\title{
SEMICLASSICAL APPROXIMATION FOR CHERN-SIMONS THEORY AND 3-HYPERBOLIC INVARIANTS
}

\author{
A.A. BYTSENKO, L. VANZO, AND S. ZERBINI
}

\begin{abstract}
The invariant integration method for Chern-Simons theory defined on the compact hyperbolic manifold $\Gamma \backslash \mathbb{H}^{3}$ is verified in the semiclassical approximation. The semiclassical limit for the partition function is presented. We discuss briefly $L^{2}$ - analytic torsion and the eta invariant of Atiyah-Patodi-Singer for compact hyperbolic 3-manifolds.
\end{abstract}

\section{INTRODUCTION}

It is known that topological invariants associated with 3-manifolds can be constructed within the framework of Chern-Simons gauge theory [1]. These values have been specified in terms of the axioms of topological quantum field theory in [2], whereas the equivalent derivation of invariants has also been presented combinatorially in [3, 4], where modular Hopf algebras related to quantum groups have been used. The Witten's (topological) invariants have been explicitly calculated for a number of 3-manifolds and gauge groups [1, 6, 7, 8, 9, 10, 11]. The semiclassical approximation for the Chern-Simons partition function may be expressed by the asymptotics for $k \rightarrow \infty$ of Witten's invariant of a 3-manifold $M$ and a gauge group $G$. Typically this expression is a partition function of quadratic functional. This asymptotics leads to a series of $C^{\infty}$ - invariants associated with triplets $\{M ; F ; \xi\}$ with $M$ a smooth homology 3 - sphere, $F$ a homology class of framings of $M$, and $\xi$ an acyclic conjugacy class of ortogonal representations of the fundamental group $\pi_{1}(M)$ [12]. In addition the cohomology $H(M ; A d \xi)$ of $M$ with respect to the local system related to $\operatorname{Ad} \xi$ vanishes.

Date: May, 1999.

We thank Prof. F.L. Williams for useful discussion. First author partially supported by a CNPq grant (Brazil), RFFI grant (Russia) No 98-02-18380-a, and by GRACENAS grant (Russia) No 6-18-1997. 
This note is an extension of the two previous papers [13, 14]. Here our aim is to use again the invariant integration method [15, 16] in its simplest form in order to evaluate the semiclassical approximation in the Chern-Simons theory. We do this analysing the partition function related to compact hyperbolic 3-manifolds $\Gamma \backslash \mathbb{H}^{3}$, where $\mathbb{H}^{3}$ is the real hyperbolic space and $\Gamma$ is a co-compact discrete group of isometries (for details see Ref. [17).

We conclude this section introducing the Witten's invariant defined by the partition function associated with a Chern-Simons gauge theory

$$
\mathfrak{W}(k)=\int \mathcal{D} A e^{i k C S(A)}, \quad k \in \mathbb{Z} .
$$

The formal integration in (1.1) is one over the gauge fields $A$ in a trivial bundle, i.e. 1 -forms on the 3 -dimensional manifold $X_{\Gamma}$ with values in Lie algebra $g$ of a gauge group $\mathfrak{G}$. The Chern-Simons functional $C S(A)$ can be considered as a function on a space of connections on a trivial principal bundle over a compact oriented 3-manifold $X_{\Gamma}$ given by

$$
C S(A)=\frac{1}{4 \pi} \int_{X_{\Gamma}} \operatorname{Tr}\left(A \wedge d A+\frac{2}{3} A \wedge A \wedge A\right) .
$$

Let $X$ be a locally symmetric Riemannian manifold with negative sectional curvature. Its universal covering $\widetilde{X} \rightarrow X$ is a Riemannian symmetric space of rank one. The group of orientation preserving isometries $\widetilde{G}$ of $\widetilde{X}$ is a connected semisimple Lie group of real rank one and $\widetilde{X}=\widetilde{G} / \widetilde{K}$, where $\widetilde{K}$ is a maximal compact subgroup of $\widetilde{G}$. The fundamental group of $X$ acts by covering transformations on $\widetilde{X}$ and gives rise to a discrete, co-compact subgroup $\Gamma \subset \widetilde{G}$ such that $X=\Gamma \backslash \widetilde{G} / \widetilde{K}$. Let $G$ be a linear connected finite covering of $\widetilde{G}$, the embedding $\Gamma \hookrightarrow \widetilde{G}$ lifts to an embedding $\Gamma \hookrightarrow G$. Let $K \subset G$ be a maximal compact subgroup of $G$, then $X_{\Gamma}=\Gamma \backslash G / K$ is a compact manifold. Let $\mathrm{g}=\mathfrak{k} \oplus \mathfrak{p}$ be a Cartan decomposition of the Lie algebra g of $G$. Let $\mathfrak{a} \subset \mathfrak{p}$ be a one-dimensional subspace and $J=K \cap G_{\mathfrak{a}}$ be the centralizer of $\mathfrak{a}$ in $K$. Fixing a positive root system of $(\mathrm{g}, \mathfrak{a})$ we have the Iwasawa decomposition $\mathrm{g}=\mathfrak{k} \oplus \mathfrak{a} \oplus \mathfrak{n}$. For $G=S O(n, 1)\left(n \in \mathbb{Z}_{+}\right), K=S O(n)$, and $J=S O(n-1)$. The corresponding symmetric space of non-compact type is the real hyperbolic space $\mathbb{H}^{n}$ of sectional curvature -1 . Its compact dual space is the unit $n$ - sphere.

Since $C S(A)$ does not contain any metric on $X_{\Gamma}$, the quantity $\mathfrak{W}(k)$ is expected to be metric independent, namely to be a (well-defined) topological invariant of $X_{\Gamma}$. Indeed, this fact has been proved in Refs. [3, 4. In the limit $k \rightarrow \infty$, the asymptotics of the Witten's invariant 
(semiclassical approximation of Eq. (1.1)), involves only a partition functions of quadratic functionals [1], namely

$$
\sum_{\left[A_{f}\right]} \exp \left(i k C S\left(A_{f}\right)\right) \int \mathcal{D} \omega \exp \left(\frac{i k}{4 \pi} \int_{X_{\Gamma}} \operatorname{Tr}\left(\omega \wedge d_{A_{f}} \omega\right)\right) .
$$

In above equation the sum is taken over representatives $A_{f}$ for each point $\left[A_{f}\right]$ in the moduli-space of flat gauge fields on $X_{\Gamma}$. In addition the $\omega$ are Lie-algebra-valued 1-forms and $d_{A_{f}}$ is the covariant derivative determined by $A_{f}$,

$$
d_{A_{f}} \omega=d \omega+\left[A_{f}, \omega\right]
$$

\section{QuAdRAtiC FUnCtional With ELLIPTIC RESOlVEnT}

Let $M$ be a compact oriented Riemannian manifold without boundary, and $n=2 m+1=\operatorname{dim} M$ is the dimension of the manifold. Let $\chi: \pi_{1}(M) \longmapsto O\left(V,\langle\cdot, \cdot\rangle_{V}\right)$ be a representation of $\pi_{1}(M)$ on real vectorspace $V$. The mapping $\chi$ determines (on a basis of standard construction in differential geometry) a real flat vectorbundle $\xi$ over $M$ and a flat connection map $\nabla_{p}$ on the space $\Omega^{p}(M, \xi)$ of differential $p-$ forms on $M$ with values in $\xi$. One can say that $\chi$ determines the space of smooth sections in the vectorbundle $\Lambda^{p}(T M)^{*} \otimes \xi$. One can construct from the metric on $M$ and Hermitian structure in $\xi$ a Hermitian structure in $\Lambda(T M)^{*} \otimes \xi$ and the inner products $\langle\cdot, \cdot\rangle_{m}$ in the space $\Omega^{m}(M, \xi)$. Thus

$$
S_{\mathcal{O}}=\langle\omega, \mathcal{O} \omega\rangle_{m}, \quad \mathcal{O}=* \nabla_{m},
$$

where $(*)$ is the Hodge-star map. The map $\mathcal{O}$ is formally self-adjoint with the property $\mathcal{O}^{2}=\nabla_{m}^{*} \nabla_{m}$. Suppose that the quadratic functional (2.1) is defined on the space $\mathcal{G}=\mathcal{G}(M, \xi)$ of smooth sections in a real Hermitian vectorbundle $\xi$ over $M$. There exists a canonical topological elliptic resolvent $R\left(S_{\mathcal{O}}\right)$, related to the functional (2.1), namely

$$
0 \stackrel{0}{\longrightarrow} \Omega^{0}(M, \xi) \stackrel{\nabla_{0}}{\longrightarrow} \ldots \stackrel{\nabla_{m-2}}{\longrightarrow} \Omega^{m-1}(M, \xi) \stackrel{\nabla_{m-1}}{\longrightarrow} \operatorname{ker}\left(S_{\mathcal{O}}\right) \stackrel{0}{\longrightarrow} 0 .
$$

Therefore, for the resolvent $R\left(S_{\mathcal{O}}\right)$, we have $\mathcal{G}_{p}=\Omega^{m-p}(M, \xi)$ and $H^{p}\left(R\left(S_{\mathcal{O}}\right)\right)=H^{m-p}(\nabla)$, where $H^{p}(\nabla)=\operatorname{ker}\left(\nabla_{p}\right) / \Im\left(\nabla_{p-1}\right)$ are the cohomology space. Note that $S_{\mathcal{O}} \geq 0$ and therefore $\operatorname{ker}\left(S_{\mathcal{O}}\right) \equiv \operatorname{ker}(\mathcal{O})=$ $\operatorname{ker}\left(\nabla_{m}\right)$.

Let us choose an inner product $\langle\cdot, \cdot\rangle_{H^{p}}$ in each space $H^{p}\left(R\left(S_{\mathcal{O}}\right)\right)$. The partition function of $S_{\mathcal{O}}$ with the resolvent (2.2) can be written in the form (see Refs. [18, 16]) 


$$
\begin{gathered}
\mathfrak{W}(k) \equiv \mathfrak{W}\left(k ; R\left(S_{\mathcal{O}}\right),\langle\cdot, \cdot\rangle_{H},\langle\cdot, \cdot\rangle\right)=\left(\frac{\pi}{k}\right)^{\zeta(0,|\mathcal{O}|) / 2} e^{-\frac{i \pi}{4} \eta(0, \mathcal{O})} \\
\times \tau\left(M, \chi,\langle\cdot, \cdot\rangle_{H}\right)^{1 / 2},
\end{gathered}
$$

where $|\mathcal{O}|=\sqrt{\mathcal{O}^{2}}$ is defined via spectral theory. This is the basic formula one has to evaluate. With regard to the quantity $\tau\left(M, \chi,\langle\cdot, \cdot\rangle_{H}\right)$, it is related the Ray-Singer torsion. In fact, if $H^{0}(\nabla) \neq 0$ and $H^{p}(\nabla)=$ 0 for $p=1, \ldots, m$, then the product

$$
\tau\left(M, \chi,\langle\cdot, \cdot\rangle_{H}\right)=T_{a n}^{(2)}(M) \cdot \operatorname{Vol}(M)^{-\operatorname{dim} H^{0}(\nabla)},
$$

is metric independent 19, i.e. the metric dependence of the Ray-Singer torsion $T_{a n}^{(2)}(M)$ factors out as $V(M)^{-\operatorname{dim} H^{0}(\nabla)}$.

As far as the zeta-function $\zeta(0,|\mathcal{O}|$ is concerned, we recall that there exists $\varepsilon, \delta>0$ such that for $0<t<\delta$ the heat kernel expansion for self-adjoint Laplace operators $\mathfrak{L}_{p}$ is given by

$$
\operatorname{Tr}\left(e^{-t \mathfrak{L}_{p}}\right)=\sum_{0 \leq \ell \leq \ell_{0}} a_{\ell}\left(\mathfrak{L}_{p}\right) t^{-l}+O\left(t^{\varepsilon}\right) .
$$

Starting with the formula 18

$$
\zeta\left(0, \mathfrak{L}_{p}\right)=a_{0}\left(\mathfrak{L}_{p}\right)-\operatorname{dim}\left(\operatorname{ker}\left(\mathfrak{L}_{p}\right)\right)=a_{0}\left(\mathfrak{L}_{p}\right)-\operatorname{dim} H^{p}(R(S)),
$$

one can shown that the zeta function $\zeta(s,|\mathcal{O}|)$ is well-defined and analytic for $\Re(s)>0$ and can be continued to a meromorphic function on $\mathbb{C}$, regular at $s=0$ and

$$
\zeta(0,|\mathcal{O}|)=\sum_{p=0}(-1)^{p}\left(a_{0}\left(\mathfrak{L}_{p}\right)-\operatorname{dim} H^{p}(R(S))\right) .
$$

Furthermore, the zeta function $\zeta(0,|\mathcal{O}|)$ appearing in the partition function (2.3) can be expressed in terms of the dimensions of the cohomology spaces of $\mathcal{O}$. Indeed, if the dimension of $M$ is odd $(n=2 m+1)$ then for all $p a_{0}\left(\mathfrak{L}_{p}\right)=0$, because we are dealing with manifold without boundary. Since $H^{p}\left(R\left(S_{\mathcal{O}}\right)\right)=H^{m-p}(\nabla)$ (the Poincarè duality) for the resolvent $(2.2)$, it follows that

$$
\zeta(0 \| \mathcal{O} \mid)=-\sum_{p=0}^{m}(-1)^{p} \operatorname{dim} H^{p}(R(S))=(-1)^{m+1} \sum_{p=0}^{m}(-1)^{p} \operatorname{dim} H^{p}(\nabla) .
$$

Finally, the dependence of the eta invariant $\eta(0 \mid \mathcal{O})$ of Atiyah-PatodiSinger on the connection map $\mathcal{O}$ can be expressed with the help of the formula for the index of the twisted signature operator for a certain 
vectorbundle over $M \otimes[0,1]$ (see [20, 21, 22]). Furthermore It can be shown [18 that

$$
\eta(s \mid B)=2 \eta(s \mid \mathcal{O})
$$

where the $B$ are elliptic self-adjoint maps on $\Omega(M, \xi)$ defined on $p$-forms by

$$
B_{p}=(-i)^{\lambda(p)}\left(* \nabla+(-1)^{p+1} \nabla *\right),
$$

In this formula $\lambda(p)=(p+1)(p+2)+m+1$ and for the Hodge star-map we have used $* \alpha \wedge \beta=\langle\alpha, \beta\rangle_{\text {vol }}$. From the Hodge theory we have

$$
\operatorname{dimker} B=\sum_{p=0}^{m} \operatorname{dim} H^{p}(\nabla) \text {. }
$$

\section{THE CASE OF REAL COMPACT HYPERBOLIC MANIFOLDS}

In this section, we shall consider the specific case of a compact hyperbolic 3-manifolds of the form $M=X_{\Gamma}=\Gamma \backslash \mathbb{H}^{3}$. If the flat bundle, $\xi$ is acyclic, then for $L^{2}-$ torsion one gets $\left[23:\left[T_{a n}^{(2)}\left(X_{\Gamma}\right)\right]^{2}=\mathfrak{R}_{\chi}(0)\right.$, where $\mathfrak{R}_{\chi}(s)$ is the Ruelle function. The function $\mathfrak{R}_{\chi}(s)$ is an alternating product of more complicate factors, each of which is a Selberg zeta function $Z_{p}(s ; \chi)$. The relation of Ruelle and Selberg functions is:

$$
\mathfrak{R}_{\chi}(s)=\prod_{p=0}^{\operatorname{dim} M-1} Z_{p}(p+s ; \chi)^{(-1)^{j}}
$$

The function $\mathfrak{R}_{\chi}(s)$ extends meromorphically to the entire complex plane $\mathbb{C}$ [24]. The Ruelle function associated with closed oriented hyperbolic 3-manifold $X_{\Gamma}=\Gamma \backslash \mathbb{H}^{3}$ has the form $\mathfrak{R}_{\chi}(s)=Z_{0}(s ; \chi) Z_{2}(2+$ $s ; \chi) / Z_{1}(1+s ; \chi)$. The analytic torsion for manifold $X_{\Gamma}$ has been calculated (in the presence of non-vanishing Betti numbers $b_{i} \equiv b_{i}\left(X_{\Gamma}\right)=$ $\left.\operatorname{rank}_{\mathbb{Z}} H_{i}\left(X_{\Gamma} ; \mathbb{Z}\right)\right)$ in Refs. [13, 14].

Now we consider the evaluation of eta invariant contribution. With regard to this point, a remarkable formula relating $\eta(s, \mathcal{O})$ to the closed geodesics on $X_{\Gamma}$ has been obtained by Millson [25]. More explicitly, Millson has proved the following result for a Selberg type (Shintani) zeta function $\widetilde{Z}(s, \mathcal{O})$.

Let us define a zeta function by the following series, which is absolutely convergent for $\Re(s)>0$,

$$
\log \widetilde{Z}(s, \mathcal{O}) \stackrel{\text { def }}{=} \sum_{[\gamma] \neq 1} \frac{\operatorname{Tr} \tau_{\gamma}^{+}-\operatorname{Tr} \tau_{\gamma}^{-}}{\left|\operatorname{det}\left(I-P_{h}(\gamma)\right)\right|^{1 / 2}} \frac{e^{-s \ell(\gamma)}}{m(\gamma)}
$$


where $[\gamma]$ runs over the nontrivial conjugacy classes in $\Gamma=\pi_{1}\left(X_{\Gamma}\right)$, $\ell(\gamma)$ is the length of the closed geodesic $c_{\gamma}$ (with multiplicity $m(\gamma)$ ) in the free homotopy class corresponding to $[\gamma], P_{h}(\gamma)$ is the restriction of the linear Poincaré map $P(\gamma)=d \Phi_{1}$ at $\left(c_{\gamma}, \dot{c}_{\gamma}\right) \in T X_{\Gamma}$ to the directions normal to the geodesic flow $\Phi_{t}$ and $\tau_{\gamma}^{ \pm}$is the parallel translation around $c_{\gamma}$ on $\Lambda_{\gamma}^{ \pm}= \pm i$ eigenspace of $\sigma_{B}\left(\dot{c}_{\gamma}\right)$ ( $\sigma_{B}$ denoting the principal symbol of $\mathcal{O})$. Then $\widetilde{Z}(z, \mathcal{O})$ admits a meromorphic continuation to the entire complex plane, which in particular is holomorphic at $s=0$ and

$$
\log \widetilde{Z}(0, \mathcal{O})=\pi i \eta(0, \mathcal{O})
$$

Furthermore, it is possible to show that $\widetilde{Z}(s, \mathcal{O})$ satisfies the functional equation

$$
\widetilde{Z}(s, \mathcal{O}) \widetilde{Z}(-s, \mathcal{O})=e^{2 \pi i \eta(0, \mathcal{O})} .
$$

Now we have all the ingredients for the evaluation of the partition function (2.3) in terms of $L^{2}$ - analytic torsion and a Selberg type function. The final result is

$$
\mathfrak{W}(k)=\left(\frac{\pi}{k}\right)^{\zeta(0,|\mathcal{O}|) / 2} \widetilde{Z}(0, \mathcal{O})^{-1 / 4}\left[T_{a n}^{(2)}\left(X_{\Gamma}\right)\right]^{1 / 2}[\operatorname{Vol}(\Gamma \backslash G)]^{-\operatorname{dim} H^{0}(\nabla) / 2}
$$

where $\zeta(0, \mathcal{O})$ and $\widetilde{Z}(0, \mathcal{O})$ are given by Eqs. (2.8) and (3.2) respectively.

\section{Concluding Remarks}

For a real compact hyperbolic 3-manifold, the formula (3.5) gives the value of the asymptotics of the Chern-Simons-Witten invariant. This is the main result of our paper. The invariant (3.5) involves the $L^{2}$ - analytic torsion, which can be expressed by means of Selberg zeta functions and a Shintani zeta function $\widetilde{Z}(0, \mathcal{O})$ associated with the eta invariant of Atiyah-Patodi-Singer 20]. Finally we note that the explicit result (3.5) can be very important for investigating the relation between quantum invariants for an oriented 3-manifold, defined with the help of a representation theory of quantum groups [3, 4], and Witten's invariant [1], which is, instead, related to the path integral approach.

\section{REFERENCES}

[1] E. Witten, Commun. Math. Phys. 121 (1989) 351.

[2] G. Moor and N. Seiberg, Commun. Math. Phys. 123 (1989) 77.

[3] N. Reshetikhin and V. Turaev, Commun. Math. Phys. 127 (1990) 1. 
[4] N. Reshetikhin and V. Turaev, Invent. Math. 103 (1991) 547.

[5] R. Dijkgraaf and E. Witten, Commun. Math. Phys. 129 (1990) 393.

[6] R. Kirby and P. Melvin, Invent. Math. 105 (1991) 473.

[7] D.S. Freed And R.E. Gompf, Commun. Math. Phys. 141 (1991) 79.

[8] L.C. Jeffrey, Commun. Math. Phys. 147 (1992) 563.

[9] S.K. Rama and S. Sen, Mod. Phys. Lett. A 8 (1993) 2285.

[10] L. Rozansky, Commun. Math. Phys. 171 (1995) 279.

[11] L. Rozansky, Commun. Math. Phys. 175 (1996) 275.

[12] S. Axelrod And I.M. Singer, J. Diff.Geom. 39 (1994) 173.

[13] A.A. Bytsenko, L. Vanzo And S. Zerbini, Nucl. Phys. B 505 (1997) 641.

[14] A.A. Bytsenko, A.E. Gonçalves and W. Da Cruz, Mod. Phys. Lett. A 13 (1998) 2453.

[15] A.S. Schwarz, Commun. Math. Phys. 64 (1979) 233.

[16] D.H. Adams, Phys. Lett. B 417 (1998) 53.

[17] A. A. Bytsenko, G. Cognola, L. Vanzo and S. Zerbini, Phys. Rep. 266 (1996) 1.

[18] D.H. Adams and S. Sen, "Partition Function of a Quadratic Functional and Semiclassical Approximation for Witten's 3-Manifold Invariant", hepth/9503095.

[19] D. RaY And I. Singer, Adv. Math. 7 (1971) 145.

[20] M.F. Atiyah, V.K. Patodi And I.M. Singer, Math. Proc. Camb. Phil. Soc. 77 (1975) 43.

[21] M.F. Atiyah, V.K. Patodi And I.M. Singer, Math. Proc. Camb. Phil. Soc. 78 (1975) 405.

[22] M.F. Atiyah, V.K. Patodi and I.M. Singer, Math. Proc. Camb. Phil. Soc. 79 (1976) 71.

[23] D. Fried, Invent. Math. 84 (1986) 523.

[24] A. Deitmar, Abh. Math. Sem. 59 (1989) 101.

[25] J.J. Millson, Ann. Math. 108 (1978) 1.

Departamento de Fisica, Universidade Estadual de Londrina, Caixa Postal 6001, Londrina-Parana, Brazil; on leave from Sankt-Petersburg State Technical University E-mail address: abyts@fisica.uel.br

Dipartimento di Fisica, Universitá di Trento, and Istituto Nazionale di Fisica Nucleare, Gruppo Collegato di Trento, Italy E-mail address: vanzo@science.unitn.it

Dipartimento di Fisica, Universitá di Trento, and Istituto Nazionale di Fisica Nucleare, Gruppo Collegato di Trento, Italy E-mail address: zerbini@science.unitn.it 